\title{
El deporte para todos en la organización de eventos deportivos. Las motivaciones de participación y el grado de satisfacción como posibles indicadores de control. El caso de la Trekorientación ${ }^{\circledR}$ Bosque de Poblet
}

\section{Sport for all in the sporting events organization. The motivations of participation and the satisfaction level as a possible measuring indicator.The case of Bosque de Poblet Trekorientación ${ }^{\circledR}$}

\author{
Estela Farías Torbidoni, Jordi Segui, Joan Fuster, Gonzalo Gil \\ INEFC-Universidad de Lleida
}

\begin{abstract}
Resumen: La proliferación de eventos deportivos locales promocionados como deporte para todos (DPT) es una realidad sobre la cual cabría realizar algunas cuestiones: ¿¿qué se entiende por DPT?, ¿qué requisitos deberían cumplir este tipo de eventos para ser considerados DPT?, ¿de qué forma se podría valorar el cumplimiento o no de estos requisitos? A partir de estas cuestiones, y tomando como caso de estudio la 8va Trekorientación ${ }^{\circledR}$ Bosque de Poblet, el presente trabajo plantea una propuesta de análisis y validación de la aplicación práctica del concepto DPT. Para la realización del presente proyecto se recurrió a la aplicación de una encuesta sobre una muestra representativa de 211 participantes, previa realización de 77 pre-encuestas. La observación y análisis de las principales características organizativas del evento también fueron otros de los imputs considerados en la realización de este estudio. Entre las principales conclusiones extraídas de este trabajo destaca la posibilidad de considerar: la diversidad de las motivaciones de participación y el alto grado de satisfacción de estas como posibles indicadores de medida aptos para comprobar y validar que un evento deportivo es DPT. Estos dos aspectos fueron identificados en los resultados.

Palabras clave: Deporte para todos, evento deportivo, diversidad de participantes, motivaciones, grado de satisfacción, Trekorientación ${ }^{\circledR}$ Bosque de Poblet.
\end{abstract}

Abstract: The increasing proliferation of sporting events promoted under the name SFA at the local level is a reality that we cannot ignore, and raises the questions: What do we understand by SFA? What requirements must be met for a sport to be considered as such? How do we could measure these requirements? In order to respond to these questions, the present paper presents a case study of the practical application of the SFA concept by the organizers of the 8th edition of Poblet Forest Trek-Orienteering. To conduct this study we applied a survey model that had already been tested in previous edition with a total of 77 surveys. In the studied edition a representative sample of 211 participants was used. The main observed features of the organization of this event were also used as inputs. Two key factors were identified that allow us to consider a sports event under the perspective of SFA: diversity of motivations for participation and degree of satisfaction. These two factors were identified in the results.

Key words: Sport for All, sports event, participant diversity, motivation, level of satisfaction, Poblet Forest Trek-Orienteering.

\section{Introducción}

Deporte para Todos (DPT) ha sido y sigue siendo la base fundamental de la dimensión social del deporte. Ya, en el año 1896 el Sr. Pierre de Coubertain, creador de los Juegos Olímpicos Modernos, defendió y atendió dicho término. La Carta Europea del Deporte para Todos, firmada en marzo de 1975, por la primera Conferencia de Ministros Europeos responsables del deporte, o el documento de Recomendaciones a favor del DPT, elaborado por la UNESCO en el año 1979, entre otros, son claros indicadores de la trascendencia, implicaciones y apoyo que este concepto recibe al amparo de un amplio grupo de administraciones gubernamentales a nivel nacional e internacional.

Asociado a la idea de deporte recreación o de esparcimiento en el tiempo libre, Leissure Sport, el concepto de DPT nació como un eslogan y una reivindicación social de las masas, ya que en el pasado solamente unas minorías tenían acceso a la práctica deportiva.

A priori y en sentido restringido, la concepción DPT coincide, sin lugar a dudas, con aquellas prácticas o actividades físico-deportivas centradas en fomentarlaparticipación sin restricciones, de forma inclusiva, para todos (García Ferrando, 2006; Green, 2006). En esta línea, son numerosas las definiciones que se pueden encontrar al respecto. Citar por ejemplo, la elaborada por el Ministerio de Desarrollo Social y Medio Ambiente, en el Programa Deporte Para Todos, que define este concepto como: toda práctica de actividades físicas y deportivas orientadas a la población en su conjunto, sin discriminación de edad, sexo, condición física, social, cultural oétnica, generadora de situaciones de inclusión, entendiendo al deporte como un ámbito propicio para el

Fecha recepción: 03-06-13- Fecha envío revisores: 05-06-13- Fecha de aceptación: 06-08-13 Consultoria Socioambiental Ecogestión

Afores $\mathrm{s} / \mathrm{n}$ - Castelldans

25154 - Lleida

estela.farias@cag.es desarrollo social. Definición que, trasladada al objeto la organización de eventos deportivos de DPT (menos de 500 participantes, por ejemplo) podría entenderse como la posibilidad de permitir la inscripción de la mayor diversidad (socio-tipo) de participantes, que no solo cantidad.

En este sentido y tras una lectura superficial del término diversidad (variedad, desemejanza, diferencia -RAE, 2010-) cabe puntualizar, desde un punto de vista genérico, que valorar esta diversidad solo en base a la variedad/diferencia del perfil de los participantes (en este caso socio-tipo: edad, sexo, nivel educativo, etc.) sería un error; no sólo porque recogería un pequeña representación de las posibles diversidades susceptibles de ser incluidas en este tipo de eventos, sino porque valorar esta diversidad sólo a partir del socio-tipo podría llevar a obviar algunos efectos contaminantes, como la moda, las particularidades de la actividad o evento. Entendiendo la moda como el producto de la confluencia de múltiples factores sociales, culturales y políticos que determinan la prevalencia de unas tendencias sobre otras en un momento dado, y particularidades de la actividad o evento como las principales características organizativas implícitas en las correspondientes reglas de juego, participación y competición.

En base a estas puntualizaciones, y teniendo en cuenta la gran dificultad de la valorar el buen traslado de este término en la realización de este tipo de eventos, nuestra propuesta recae en la opción de considerar la diversidad de las motivaciones de participación y el alto grado de satisfacción de estas motivaciones como posibles indicadores de medida en relación a la diversidad, inclusión, y en última instancia, cumplimiento de algunos de los preceptos claves del concepto DPT. Al respecto, dos son los objetivos planteados en la realización de este trabajo: 1) valorar la adecuación o no de las motivaciones de participación y el grado de satisfacción de estas como posibles indicadores de diversidadinclusión y 2) determinar a partir de la comprobación de estas premisas si la Trekorientación ${ }^{\circledR}$ Bosque de Poblet cumple o no con los requerimientos aquí establecidos respecto al correcto traslado del concepto de DPT. En este sentido, nuestra hipótesis es que la Trekorientación ${ }^{\circledR}$ 
Bosque de Poblet si cumple con estos dos preceptos y, en consecuencia, puede ser considerado como un evento deportivo de DPT: diversidad de motivaciones de participación y alto grado de satisfacción.

\section{El caso de estudio: la Trekorientación ${ }^{\circledR}$ Boque de Poblet}

Trekorientación ${ }^{\circledR}$ es una marca registrada de una actividad físicodeportiva que combina el senderismo con algunas técnicas de orientación deportiva, siendo también una prueba deportiva reconocida y homologada por la Federación Catalana de Carreras de Orientación. La actividad consiste en que el participante encuentre el mayor número de balizas indicadas en un mapa para así concluir un itinerario preestablecido por la organización. El objetivo deportivo consiste en poder concluir el itinerario en el menor tiempo posible.

Según sus organizadores, Trekorientación ${ }^{\circledR}$ es una iniciativa basada en el respeto de las individualidades de los participantes (actividad no excluyente), aplicando estrategias y acciones que favorecen, según su dilatada experiencia (en el 2012 se celebró su décima edición consecutiva) la inclusión.

Como características principales la Trekorientación ${ }^{\circledR}$ Bosque de Poblet:

- Contempla la opción de cuatro recorridos: Verde (4-6 Km), Azul (10-12 Km), Amarillo (15-17 Km), Rojo (22-24 Km).

- Permite participar en diferentes categorías: individual (masculina o femenina), grupos (amigos o familia); siendo opcional la posibilidad de participar en ambas con o sin mascota.

-Admite la participación sin disponer de licencia federativa deportiva.

- Adapta el recorrido más corto a colectivos con necesidades especiales, salvando barreras y poniendo a disposición del participante la compañía de un monitor-guía.

- Contempla una salida diferente para cada recorrido, disponiendo de una franja de hora y media para que cada participante empiece cuando mejor le convenga.

- Imparte, de forma previa al inicio de la prueba, un taller práctico sobre el funcionamiento de la brújula y la orientación con mapa.

- Además, dispone de un reglamento específico que contempla aspectos tales como: Todo participante durante la prueba, en todo momento tiene que ceder el paso a los «Trekers» con un ritmo más rápido; al mismo tiempo que: Todo participante tiene la obligación de asistir a otro participante de la Trekorientación ${ }^{\circledR}$ con problemas.

\section{Metodología}

Para la elaboración del modelo de encuesta utilizado se recurrió a la consulta de diversas fuentes bibliográficas como Alexandris, Tsorbatzoudis y Grouios (2002); Ntoumani y Ntoumanis (2006); Vlachopoulos, Karageorghis y Terry (2000), entre otros. En total 17 fue el número de preguntas incluidas en el modelo de encuesta utilizado, validado, en este caso, a partir de la realización y análisis de 77 encuestas en la edición del año anterior (pre-encuesta). En la tabla 1 se puede consultar un detalle de las principales dimensiones y variables consideradas, como así también, al final de este trabajo el modelo de encuesta utilizado.

Téngase en cuenta en este sentido, que a pesar que la mayor parte de las preguntas incluidas en el modelo de encuesta fueron cerradas y de respuesta múltiple, en el caso de las preguntas correspondientes a motivaciones de participación, el participante tenía la opción de elegir y puntuar, dentro de una escala del 1 (no importante) al 5 (muy importante), hasta tres motivaciones principales de participación, siendo la pregunta sobre la justificación, de no satisfacción de los requerimientos físicos del recorrido de participación, la única pregunta abierta considerada en el modelo de encuesta utilizado.

Finalmente 211 fueron el número total de encuestas realizadas, correspondientes a un nivel de confianza 95.5\% y margen de error de error del 5\%. El trabajo de campo se desarrolló durante la jornada del evento. Las encuestas fueron realizadas en la zona de meta según llegaban los participantes. El criterio de selección utilizado fue aleatorio estratificado, siendo el sistema de estratificación considerado el número de grupo inscritos en cada tipo de recorrido: verde, azul, amarillo y rojo (Tabla 2).

\begin{tabular}{|c|c|c|c|}
\hline Dimensiones & \multicolumn{3}{|c|}{ Variables } \\
\hline Perfil genérico participantes & \multicolumn{3}{|c|}{$\begin{array}{l}\text { Edad, género, lugar de residencia, dedicación profesional, estudios más altos } \\
\text { alcanzados, conocimientos zona (Bosque de Poblet y oferta turistica de la } \\
\text { zona). }\end{array}$} \\
\hline Hábitos deportivos & \multicolumn{3}{|c|}{$\begin{array}{l}\text { Categońa de participación, práctica de actividad físico-deportiva, frecuencia } \\
\text { de práctica, tipología de práctica (vinculada o no a la Treko, frecuencia de } \\
\text { participación en este tipo de eventos, frecuencia participación en la } \\
\text { Trekorientación@ Bosque de Poblet. }\end{array}$} \\
\hline $\begin{array}{l}\text { Motivaciones y Grado de } \\
\text { satisfacción }\end{array}$ & \multicolumn{3}{|c|}{$\begin{array}{l}\text { Motivaciones de participación, grado de satisfacción respecto a las } \\
\text { motivaciones prioritanias y en relación a los requerimientos físicos in herentes } \\
\text { a la categoría de participación, justificación en caso de insatisfacción. }\end{array}$} \\
\hline \multicolumn{4}{|c|}{ Tabla 2. Distribución número de encuestas } \\
\hline Recorridos & $\mathrm{N}^{\circ}$ grupos inscritos & Porcentajes & $\mathbf{N}^{\circ}$ encuestas realizad as \\
\hline Verde $(4-6 \mathrm{~km})$ & 78 & 23 & 48 \\
\hline $\operatorname{Azul}(12-14 \mathrm{~km})$ & 135 & 40 & 85 \\
\hline Amarillo (16-18) & 96 & 28 & 59 \\
\hline Rojo (22-26 km) & 32 & 9 & 19 \\
\hline Total & 341 & 100 & 211 \\
\hline
\end{tabular}

\section{Análisis estadístico}

El análisis de los datos se realizó en base a análisis estadísticos descriptivos, programa SPSS (Versión 15.0). La prueba Anova de 1 Factor (Análisis de la Varianza) se utilizó para establecer las diferencias significativas entre la categoría de participación (recorridos) y el nivel de importancia atribuidos a las diferentes motivaciones de participación.

\section{Resultados}

\section{Perfil genérico de los participantes}

En términos generales destaca la existencia de un perfil medio de participantes caracterizado por: ser varón (61\%), tener entre 20-39 años (70\%), residir de forma habitual en las provincias de Tarragona y Barcelona (47\% y $30 \%$ ), ser trabajador por cuenta ajena (52\%), poseer en un nivel de estudios universitarios (70\%) y tener un buen nivel de conocimientos previos de la zona (si conocía el Bosque de Poblet de forma previa a la participación del evento, 74\%) y de la oferta turística de la comarca (si conocía la existencia de la Ruta del Cister, 83\%).

\begin{tabular}{|c|c|c|c|}
\hline Variables & Porcentaje & Variables & Porcentaje \\
\hline Género & & Dedicación labo ral & \\
\hline Hombres & 61 & Empleado por cuenta ajena & 52 \\
\hline Edad & & Funcionario & 22 \\
\hline 50 a 60 años & 5 & Autónomo & 12 \\
\hline 40 a 49 años & 18 & Estudiante & 8 \\
\hline 30 a 39 años & 47 & Estudios & \\
\hline 20 a 29 años & 23 & Universitarios & 70 \\
\hline Lugar de residencia & & Formación profesional & 15 \\
\hline Tarragona & 47 & Nivel de conocimi entos & \\
\hline Barcelona & 30 & Bos que de Poblet & 74 \\
\hline Lleida & 21 & Ruta del Cister & 83 \\
\hline \multicolumn{4}{|l|}{ Tabla 4. Hábitos deportivos } \\
\hline Variables & Porcentaje & Variables & Porcentaje \\
\hline \multicolumn{2}{|l|}{ Práctica AF forma habitual } & \multicolumn{2}{|l|}{ Frecuencia tipo eventos } \\
\hline $\mathrm{Si}$ & 89 & Siempre que puedo & 38 \\
\hline Frecuencia de práctica & & Primera vez & 22 \\
\hline Entre 1 y 2 veces semana & 34 & Puntualmente & 17 \\
\hline Entre 3 y 4 veces semana & 36 & Casi nunca & 10 \\
\hline Más de 4 veces semana & 19 & \multicolumn{2}{|c|}{ Participación Trekorientación ${ }^{\circledR}$} \\
\hline Tipo de práctica & & Primera vez & 52 \\
\hline Vinculada a la Treko & 68 & Entre dos y tres veces & 29 \\
\hline No vinculad a a la Treko & 22 & Entre cuatro y cinco veces & 14 \\
\hline
\end{tabular}

\section{Hábitos deportivos y participación en este tipo de eventos}

En términos específicos, respecto a los hábitos deportivos de los participantes, los resultados obtenidos mostraron la presencia de un elevado nivel de hábitos deportivos al responder, el 89\% de los participantes encuestados, de forma positiva a la pregunta: ¿suele practicar algún tipo de actividad físico-deportiva de forma habitual? En este sentido, destaca el hecho que mientras que más del $55 \%$ de estos practicantes declararon que suelen realizar algún tipo de práctica deportiva con una frecuencia de entre 3 y 7 veces a la semana, más del 68\% de estos destacaron el hecho de practicar algún tipo de práctica relacionadas con la actividad de la Trekorientación ${ }^{\circledR}$ (excursionismo, running, orientación, etc.). Al respecto, destaca también el hecho que mientras 
que más del 38\% de los participantes encuestados manifestó participar en este tipo de eventos Siempre que puede, el 50\% se declaró como primerizo en la participación de la Trekorientación ${ }^{\circledR}$ Bosque de Poblet. Tabla 4.

\section{Motivaciones de participación y grado de satisfacción}

Por un lado, tal y como se puede apreciar en la tabla 5 , mientras que cinco de las diez motivaciones de participación son seleccionadas por más del 40\% de los participantes: Pasar un buen rato (81.5\%), Estar con la familia-amigos (62.5\%), Acercarme al medio natural (59.7\%), Disfrutar del paisaje (47.7\%) y Hacer alguna actividad físico-deporti$v a$ (43.6\%); seis de las diez motivaciones de participación valoradas presentan diferencias significativas entre los diferentes grupos (Sig. P > .05): Pasar un buen rato (valor mínimo 4.21 grupo rojo y valor máximo 4.75 grupo azul), Entrenar para otros eventos (mínimo 1.88 grupo verde y máximo 3.58 grupo rojo), Hacer actividad físico deportiva (mínimo 3.35 grupo verde y máximo 4.21 grupo rojo), Estar con la familia - amigos (valor mínimo 2.89 grupo rojo y valor máximo 4.75 grupo verde) y Ponerme a prueba (valor mínimo 2.67 grupo verde y valor máximo 3.89 grupo rojo); poniendo en evidencia que no todos los participantes concurren a la prueba buscando lo mismo. En este sentido destaca también la presencia de una cierta correlación negativa según se observen los grupos (nivel de dificultad de la categoría seleccionada: de menor dificultad -verde - a mayor dificultad-rojo-) en relación a aspectos más de tipos sociales, o correlación positiva, según se observen los grupos (también en relación a la dificultad de la categoría de participación) respecto a la valoren aspectos más de tipo deportivos y competitivos.

\begin{tabular}{|c|c|c|c|c|c|c|c|}
\hline \multirow[b]{2}{*}{ Motivaciones participación } & \multirow[b]{2}{*}{$\begin{array}{l}\text { Priorización } \\
\text { Motivación* }\end{array}$} & \multicolumn{6}{|c|}{ Valoración atribuida** } \\
\hline & & $\begin{array}{c}\text { Valor medio } \\
\text { muestra }\end{array}$ & Verde & Azul & Amarillo & Rojo & $\begin{array}{l}\text { Sig. } \\
\mathrm{F}^{* * * *}\end{array}$ \\
\hline Acercarme al medio natural & $59.7 \%(n=126)$ & 4.24 & 4.33 & 4.25 & 4.15 & 4.26 & .906 \\
\hline Pasar un buen rato & $81.5 \%(n=172)$ & 4.63 & 4.71 & 4.75 & 4.53 & 4.21 & .026 \\
\hline Estar con la familia - amigos & $62.5 \% \quad(n=132)$ & 4.29 & 4.75 & 4.51 & 4.07 & 2.89 & .000 \\
\hline Disfrutar del paisaje & $49.7 \%(n=105)$ & 4.35 & 4.38 & 4.46 & 4.24 & 4.16 & .420 \\
\hline Entrenar para otros eventos & $14.7 \% \quad(n=31)$ & 2.44 & 1.88 & 2.36 & 2.63 & 3.58 & .000 \\
\hline $\begin{array}{l}\text { Hacer actividad físico- } \\
\text { deportiva }\end{array}$ & $43.6 \% \quad(n=92)$ & 3.92 & 3.35 & 4.06 & 4.08 & 4.21 & .000 \\
\hline Vivir nuevas experiencias & $31.7 \% \quad(\mathrm{~N}=67)$ & 3.70 & 4.04 & 3.69 & 3.51 & 3.47 & 124 \\
\hline Conocer nuevos lugares & $20.4 \% \quad(n=43)$ & 3.50 & 3.80 & 3.48 & 3.49 & 3.22 & .798 \\
\hline Poneme a prueba & $18.0 \% \quad(n=38)$ & 3.13 & 2.67 & 3.11 & 3.31 & 3.89 & .009 \\
\hline Competir & $8.5 \% \quad(n=18)$ & 2.22 & 1.83 & 2.25 & 2.34 & 2.94 & 045 \\
\hline
\end{tabular}

* Selección motiv

**Valor obtenido de la puntuación asignada por cada participante en relación al grado de importancia atribuido a cada motivación. Escala de importancia: 1, valor mín., 5 valor máx

*** Análisis dev varianza: Anova de 1 factor . Sig. $\mathrm{p}>.05$.

Por otro lado, y en relación al grado de satisfacción manifiesto respecto a las motivaciones de participación seleccionadas, los resultados afloran la existencia de un grado de satisfacción muy elevado, al superar, en el 80\% de los casos, el 95\% de satisfacción (Tabla 6).

Finalmente, y en cuanto a la satisfacción manifiesta respecto a los

\begin{tabular}{lccc} 
Tabla 6 Grado de satisfacción & & & \\
\hline Motivaciones participación & Priorización* & $\begin{array}{c}\text { Valoración } \\
\text { positiva*** }^{*}\end{array}$ & $\begin{array}{c}\text { Nivel de } \\
\text { satisfacción*** }\end{array}$ \\
\hline Acercame al medio natural & 126 & 120 & $95.2 \%$ \\
Pasarun buen rato & 172 & 164 & $95.3 \%$ \\
Entrenar para otros even tos & 31 & 28 & $90.3 \%$ \\
Hacer actividad físico-dep ortiva & 92 & 88 & $95.6 \%$ \\
Estar con la familia - amigos & 132 & 129 & $97.7 \%$ \\
Vivir nuevas experiencias & 67 & 64 & $95.5 \%$ \\
Conocer nuevos lugares & 43 & 38 & $\mathbf{8 8 . 3 \%}$ \\
Ponerme a prueba & 38 & 33 & $\mathbf{8 6 . 8 \%}$ \\
Competir & 18 & 17 & $94.4 \%$ \\
Disfrutar del pais aje & 105 & 101 & $96.2 \%$ \\
\hline
\end{tabular}

$\mathrm{N}=211$

* Número de individuos que selecciona la motivación como prioritaria.Posibilidad de seleccionar hasta

tres motivaciones.

** Número de individuos que valora como muy satis fecho o satisfecha el cu mplimi ento de la motivación seleccionada

*** Porcentaje de satisfacción respecto a las motivaciones seleccionadas. requerimientos físicos necesarios en la categoría de participación, más del $97 \%$ de los participantes la valoran como Muy correcto y correcto. Sólo el 3\% manifestó no estar satisfecho, de los cuales 3 personas justificaron su insatisfacción porque les falto hidratación y otras 3 porque iban con gente más entrenada o se había apuntado a una categoría inadecuada a sus capacidades físicas. En la figura 2 se puede observar también como son los participantes inscritos en el recorrido más corto (verde) y más largo (rojo) los que presentan un mayor grado de satisfacción al respecto, superando la valoración de Muy correcto, se adecuó perfectamente, a la de Correcto, se adecuó bastante.

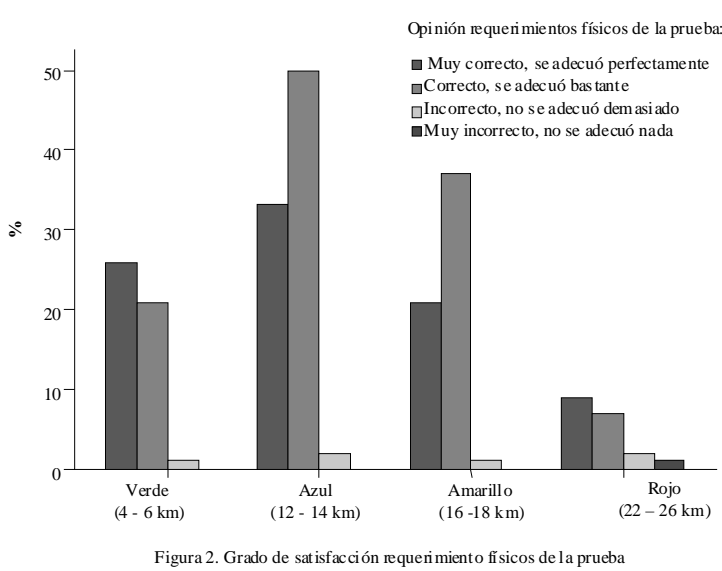

Discusión

En base al posicionamiento del concepto DPT, los resultados obtenidos confirman que considerar como indicador único la diversidad de perfiles de participantes (socio-tipo) puede no ser suficiente, ya que si nos remitiéramos a los resultados obtenidos en este estudio solo considerando el socio-tipo (sexo, edad, nivel educativo, etc.) de los participantes como indicador de diversidad, las conclusiones obtenidas serian que la Trekorientación ${ }^{\circledR}$ Bosque de Poblet no cumple los requisitos de diversidad o inclusión comprendidos dentro del concepto de DPT, al no observarse una suficiente heterogeneidad en el socio-tipo de los participantes.

En este sentido, se ha de tener en cuenta que podemos encontrar perfiles de participantes homogéneos con motivaciones diferentes y viceversa. Deaquí, que considerar la diversidad, como aspecto delimitador del término DPT, debería venir justificado por otro tipo de indicadores como los propuestos en este trabajo: diversidad de las motivaciones de participación y grado de satisfacción de las mismas. Aspectos que analizados de forma complementaria pueden avalar, aunque no de forma totalitaria, que un evento deportivo si pude ser considerado como DPT.

En este punto cabe destacar la trascendencia que en este sentido puede tener la satisfacción de las motivaciones por su carácter verificador. Así, la presencia de una amplia diversidad de motivaciones y un bajo nivel de satisfacción o la presencia de una escasa o nula diversidad de motivaciones y un alto grado de satisfacción dejaría en entredicho la consecución real del objetivo de DPT.

Finalmente, y trasladando las conclusiones teóricas en aplicaciones prácticas, dos pueden ser los consejos básicos a seguir por toda organización que organice eventos de DPT:

- Permitir la inscripción de una amplia diversidad de participantes, incluidas la inclusión de sus múltiples motivaciones. No confundirse con obtener un gran número de participantes. La organización ha de ser sensible, en la medida que los condicionantes externos (contexto sociopolítico y actividad físico-deportiva) lo permitan, a atender la mayor diversidad de tipologías de participantes siempre: siendo conscientes del esfuerzo organizativo que este hecho implica.

- Lograr el máximo grado de satisfacción de las motivaciones. Para ello quizás resulte necesario intentar recoger en el momento de la inscripción, las motivaciones de participación, dando margen al organiza- 
dor a valorar la adecuación de la prueba a los diferentes perfiles, como así también para ayudar a reflexionar en la mejora continua de las futuras ediciones.

\section{Referencias}

Alexandris, K., Tsorbatzoudis, C., \& Grouios, G. (2002). Perceived Constraints on Recreational Sport Participation: Investigating Their Relationship with Intrinsic Motivation, Extrinsic Motivation and Amotivation. Journal of Leisure Research, 34(3), 233-52.

García Ferrando, M. (2006). Encuesta de hábitos deportivos de los Españoles 2005.Posmodernidad y Deporte: Entre la Individualización y la Masificación. Consejo Superior de Deportes. Madrid.

Green, M. (2006). From Sport for All' to Not About 'Sport' at All?: Interrogating

Sport Policy Interventions in the United Kingdom (2006). European
Sport Management Quarterly. 6(3), 217-238.

Dechavanne, N. (1991). El animador de las actividades físicos deportivas para todos. Barcelona: Paidos.

Ntoumani, C.T., \& Ntoumanis, N.: (2006). The role of self-determined motivation in the understanding of exercise-related behaviours, cognitions and physical self-evaluation. Journal of Sports Sciences, 24, 393-404.

Real Academia Española. Diccionario de la Lengua Española - Vigésima segunda edición

Vlachopoulos, S.P., Karageorghis, C.I., \& Terry, P.C. (2000). Motivation profiles in sport: A Self-determination Theory perspective. Research Quarterly for Exercise and Sport , 71, 387-97.

Agradecimientos: Agradecer a los alumnos que colaboraron en la realización del trabajo de campo: Cristian Montoro, Eva Higuera, Elias Borras, Mikel Oriols, Joan Casas, Joel Piñero, Adrià Martinez, Pau Fernandez, Jonathan Vidal y Joan Serra.

\section{Modelo de Encuesta utilizado}

Hora: Lugar: Encuestador/a: Número dorsal:

I- L ugar de residencia habi tual

1- Barcelona. Especificar lugar

2- Lleida. Especificar lugar

3- Tarragona. Especificar lugar

4-Girona. Especificar lugar

5- Otras provin cias

6- Otros países

II-Dedicación laboral - profesional

1- Estudiante

2- Jubi lado(a) pensionista

3-Parado a)/desocupado(a)

4- Ama de cas a

5- Funcionario(a)

6- Empl eado(a) por cuent a propia o autónomo

7-Empl eado(a) por cuent a de otro

8-Empresario

\section{V-Año de nacimiento}

VI- In dicar frecuencia actividad deportiva

1- Practico actividad física de forma habitual

2- No practico actividad física de forma habitual

VII- En cas afirmativo indicar frecuencia global

1-2-3-4-5-6-7 días a la semana

VIII- Especificar tipo/s de actividad/es

Física/s que suele practicar de forma habitual.

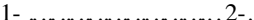

IX-Frecuencia en la participación de es te tipo de eventos

1- Siempre que puedo

2- A veces, en función del tipo de evento

3- Puntualmente

4- Casi nunca

X-Frecuencia de participación Trekoorientación Bo sque de Poblet In dicar veces: $1,2,3,4,5,6,7,8,9,10$

XI- Recorrido en el que se ha inscrito

1- Verde $(4-6 \mathrm{~km})$

2- Azul (12-14 km)

3- Amarillo (16- $18 \mathrm{~km})$

4- Rojo (22- $26 \mathrm{~km})$

1- Hombre

2-Mujer

XII - ¿Sabia Ud.que el bosque de Poblet es un Es pacio Natural protegido?

XIII - Antes de partici par en este evento, ¿conocía la zona: Ru ta del Cister, por ejemplo?

XIV-Motivos por los cuales ha partici pado en este even to. Puntuar del 1 al 5 (1: menos importante - 5: más

importante) según su o rden de preferencia

1- Para acercame al medio $n$ atural

2- Para pasar un buen rato

3- Para entrenar de cara a otros eventos

4- Para hacer actividad físico-esportiva

5- Para estar con la famíli a o amigos

6- Para vivir nuevas experi encias

7- Para conocer nuevos lugares

8- Para ponerme a prueba

9- Para competir

10-Disfrutar del paisage, etc

11-Otras. Especificar

XV- De los aspectos de la pregunta anterior que has puntuado con un 4 o un 5, indiquenos, por favor, su nivel de satisfacción en relacion a sus expectativas

(1 muy ins ati sfecho - 5 muy satisfecho)

1- Aspecto 1

2- Aspecto 2

3-Aspecto 3

XVI- ¿Que opi nón le merecen las capacidades físicas requeri das en la categoría que ha participado?

1- Muy correcta (se adecuó perfectamente a mi/nuestras capacidades físicas - expectativas)

2-Correcta (se adecuó bast ante a mi/nues tras capacidades físicas - expect ativas )

3- Incorrecta (no se adecuó d emasiado a mi/nuestras capacidades físi cas - expectativas)

4- Muy incorrect a (No se adecuó nada a mi/nuestras capacidades físicas - expectativas)

XVII- En caso de haber respondido con un 3 o 4 de la pregunta anterior, indique el porqué?

Especificar 